\title{
Statins as antioxidant therapy for preventing cardiac myocyte hypertrophy
}

\author{
Masao Takemoto, ${ }^{1}$ Koichi Node, ${ }^{2}$ Hironori Nakagami, ${ }^{1}$ Yulin Liao, ${ }^{2}$ Michael Grimm, ${ }^{1}$ \\ Yaeko Takemoto, ${ }^{1}$ Masafumi Kitakaze, ${ }^{2}$ and James K. Liao ${ }^{1}$ \\ ${ }^{1}$ Vascular Medicine Unit, Cardiovascular Division, Brigham and Women's Hospital and Harvard Medical School, Boston, \\ Massachusetts, USA \\ ${ }^{2}$ Department of Internal Medicine and Therapeutics, Osaka University Graduate School of Medicine, Osaka, Japan \\ Address correspondence to: James K. Liao, Vascular Medicine Unit, Brigham and Women's Hospital, \\ 221 Longwood Avenue, LMRC-322, Boston, Massachusetts 02115, USA. \\ Phone: (617) 732-6538; Fax: (617) 264-6336; E-mail: jliao@rics.bwh.harvard.edu.
}

Received for publication May 23, 2001, and accepted in revised form September 24, 2001.

\begin{abstract}
Cardiac hypertrophy is a major cause of morbidity and mortality worldwide. The hypertrophic process is mediated, in part, by small $\mathrm{G}$ proteins of the Rho family. We hypothesized that statins, inhibitors of 3-hydroxy-3-methylglutaryl-CoA reductase, inhibit cardiac hypertrophy by blocking Rho isoprenylation. We treated neonatal rat cardiac myocytes with angiotensin II (AngII) with and without simvastatin $(\mathrm{Sim})$ and found that Sim decreased AngII-induced protein content, $\left[{ }^{3} \mathrm{H}\right]$ leucine uptake, and atrial natriuretic factor $(A N F)$ promoter activity. These effects were associated with decreases in cell size, membrane Rho activity, superoxide anion $\left(\mathrm{O}_{2} \cdot^{-}\right)$production, and intracellular oxidation, and were reversed with L-mevalonate or geranylgeranylpyrophosphate, but not with farnesylpyrophosphate or cholesterol. Treatments with the Rho inhibitor C3 exotoxin and with cell-permeable superoxide dismutase also decreased AngII-induced $\mathrm{O}_{2} \cdot{ }^{-}$production and myocyte hypertrophy. Overexpression of the dominant-negative Rho mutant N17Rac1 completely inhibited AngII-induced intracellular oxidation and $A N F$ promoter activity, while N19RhoA partially inhibited it, and N17Cdc 42 had no effect. Indeed, Sim inhibited cardiac hypertrophy and decreased myocardial Rac1 activity and $\mathrm{O}_{2}{ }^{-}$production in rats treated with AngII infusion or subjected to transaortic constriction. These findings suggest that statins prevent the development of cardiac hypertrophy through an antioxidant mechanism involving inhibition of Rac1.
\end{abstract}

J. Clin. Invest. 108:1429-1437 (2001). DOI:10.1172/JCI200113350.

\section{Introduction}

Cardiac hypertrophy represents an initial physiological adaptive response to increases in blood pressure or afterload (1). However, despite normalization of systemic blood pressure by medication, cardiac hypertrophy frequently decompensates into congestive heart failure (2). Indeed, cardiac hypertrophy is an independent risk factor in cardiovascular disease, and increases cardiovascular mortality by more than twofold (3). The hypertrophic process is characterized by the induction of immediate-early genes such as $c-f o s$, $c-j u n$, and erg-1; the reexpression of embryonic genes such as atrial natriuretic factor (ANF), $\beta$-myosin heavy chain, and skeletal $\alpha$-actin (4); and the increased synthesis of contractile proteins such as myosin light chain-2 (MLC-2v) and cardiac $\alpha$-actin $(5,6)$.

Stimulation of the angiotensin II (AngII) type I receptor or exposure to pressure overload induces a cardiac hypertrophic response mediated in part by the activation of the heterotrimeric $G$ proteins (e.g., $\left.G_{q}\right)(7,8)$ and small $G$ proteins (e.g., Ras and Rho) (9-11). In particular, the Rho proteins, RhoA, Rac1, and Cdc42, are thought to play critical roles in the hypertrophic process by regulating cell morphology and contractile elements
(12). For example, previous studies have shown that RhoA is required for $G \alpha_{q}$ and $\alpha_{1}$-receptor signaling in cardiac myocytes, and that dominant-negative mutants of RhoA attenuate cardiac myocyte hypertrophy in response to stimulation with phenylephrine $(11,13)$. Surprisingly, cardiac-specific overexpression of RhoA in mice does not lead to cardiac hypertrophy, but produces sinus and atrioventricular nodal dysfunction and development of ventricular failure (14). These results suggest that other pathways may contribute to the hypertrophic response. Indeed, Rac1, and not RhoA, is required for signal transduction pathways leading to cardiac myocyte hypertrophy (15).

Current treatments for cardiac hypertrophy are limited to vasodilators or afterload reducers, with few if any therapies directed at the myocardial process. The 3-hydroxy-3-methylglutaryl-CoA (HMG CoA) reductase inhibitors, or statins, are widely prescribed cholesterol-lowering agents that decrease the incidence of myocardial infarction and ischemic stroke $(16,17)$. In addition to inhibiting cholesterol synthesis, the statins also inhibit the synthesis of important isoprenoid intermediates that are important lipid attachments required for the subcellular localization and function 
of a variety of proteins, including the Rho proteins (18, 19). The purpose of this study, therefore, was to determine whether statins can attenuate cardiac hypertrophy by inhibiting Rho proteins, and if so, to determine the potential underlying mechanism involved.

\section{Methods}

Cardiac myocyte culture. We obtained ventricles from 1day-old Sprague-Dawley rats, and isolated cardiac myocytes by digestion with trypsin-EDTA and type 2 collagenase as described (20). Cells were cultured in serum-free insulin-transferrin (IT) medium (21) for an additional 24-36 hours. Using this method, we obtained primary cultures of greater than $95 \%$ cardiac myocytes, as assessed by microscopic observation of spontaneous cellular contractions and immunofluorescence staining of anti-cardiac myosin heavy chain. Unless otherwise indicated, all reagents were obtained from Sigma Chemical Co. (St. Louis, Missouri, USA), and were used at the following final concentrations: AngII, $1 \mu \mathrm{M}$; preactivated simvastatin (Sim; Brigham \& Women's Hospital Pharmacy), 0.1-10 $\mu \mathrm{M}$; L-mevalonate (L-mev), $200 \mu \mathrm{M}$; farnesylpyrophosphate (FPP), $10 \mu \mathrm{M}$; geranylgeranylpyrophosphate (GGPP), $10 \mu \mathrm{M}$; LDL cholesterol, $1 \mathrm{mg} / \mathrm{ml}$; farnesyltransferase inhibitor (FTI276; Calbiochem-Novabiochem Corp., San Diego, California, USA), $20 \mathrm{nM}$; geranylgeranyl transferase inhibitor (GGTI-286; Calbiochem-Novabiochem Corp.), $30 \mu \mathrm{M}$; Clostridium botulinum C3 transferase (C3 TF; List Biological Laboratories Inc., Campbell, California, USA), $50 \mu \mathrm{g} / \mathrm{ml}$; polyethylene glycol-conjugated superoxide dismutase (PEG-SOD), $50 \mathrm{U} / \mathrm{ml}$; polyethylene glycol-conjugated catalase (PEG-CAT), $500 \mathrm{U} / \mathrm{ml}$; and polyethylene glycol (PEG), $0.32 \mathrm{mg} / \mathrm{ml}$. The PEG, PEG-SOD, and PEG-CAT were dissolved in IT medium and incubated with cardiac myocytes for 1 hour before AngII stimulation. Cardiac myocytes were stimulated for 24 hours. Viability was determined by cell number, frequency of contractions (i.e., intrinsic heart rate), cellular morphology, and trypan blue exclusion.

Measurements of cardiac bypertrophy. We measured $\left[{ }^{3} \mathrm{H}\right]$ leucine uptake as described (22). Cardiac myocytes were incubated for 36 hours in serum-free IT medium, then treated under the indicated conditions in the presence of diluent or $\left[{ }^{3} \mathrm{H}\right]$ leucine $(1 \mu \mathrm{Ci} / \mathrm{ml})$ for 24 hours. After incubating at room temperature for 45 minutes, cellular proteins were precipitated with $5 \%$ trichloroacetic acid and resuspended in $0.4 \mathrm{~N} \mathrm{NaOH}$, and the radioactivity was counted in a scintillation counter (Beckman LS 6000IC; Beckman Instruments Inc., Fullerton, California, USA).

Northern blotting. Equal aliquots of total RNA $(15 \mu \mathrm{g})$ were separated by $1 \%$ formaldehyde-agarose gel electrophoresis, and hybridization and washing were performed as previously described (23). The ANF and MLC-2 $\mathrm{v}$ cDNAs were labeled with random hexamer primer using $\left[\alpha-{ }^{32}\right.$ P]CTP (NEN Life Science Products Inc., Boston, Massachusetts, USA) and Klenow (Amersham Pharmacia Biotech Inc., Piscataway, New Jersey,
USA). Loading conditions were determined by ethidium bromide staining of 28S ribosomal RNA. Blots were analyzed by laser densitometry.

Transient transfections. Nearly confluent rat cardiac myocytes were transfected with the indicated cDNAs using the calcium-phosphate coprecipitation method (24). The following amounts of cDNAs were used: mutant c-myc-tagged RhoA, Rac1, and Cdc42 (10 $\mu \mathrm{g}$ each); ANF-Luc reporter plasmid $(15 \mu \mathrm{g})$; and $\beta$-galactosidase plasmid $(4 \mu \mathrm{g})$. Approximately 48 hours after transfection, the myocytes were treated as indicated for an additional 24 hours. Luciferase activity was normalized to $\beta$-galactosidase activity for each sample, to correct for variations in transfection efficiency. Results are expressed as relative ratio of control (fold induction). Preliminary studies indicate that the transfection efficiency was approximately $2-4 \%$.

Immunofluorescence. Following transfection and stimulation under the indicated conditions, rat cardiac myocytes were treated with $10 \mu \mathrm{g} / \mathrm{ml}$ of dichlorofluorescin-diacetate (Molecular Probes Inc., Eugene, Oregon, USA) for 1 minute, then fixed with $1 \%$ paraformaldehyde and permeabilized with $0.1 \%$ Triton $\mathrm{X}-100$. For staining of the $\mathrm{c}-\mathrm{myc}$ tag, cells were incubated with mouse anti-human c-myc antibody (1:100 in 1\% BSA; Transduction Laboratories, Lexington, Kentucky, USA) at $37^{\circ} \mathrm{C}$ for 20 minutes. After cells were washed with $1 \%$ BSA, R-phycoerythrin-conjugated goat antmouse IgG (red fluorescence) was used as secondary antibody (1:100 in 1\% BSA). For staining of $\alpha$-actinin and desmin, cells were incubated with a mouse monoclonal antibody to $\alpha$-actinin (1:200 in 1\% BSA) and a rabbit polyclonal antibody to desmin (1:100 in 1\% BSA; Sigma Chemical Co.). After cells were washed with $1 \%$ BSA in PBS, they were treated with these secondary antibodies: FITC-conjugated goat anti-mouse $\operatorname{IgG}(\mathrm{H}+\mathrm{L})$ (green fluorescence) and TRITC-conjugated anti-rabbit IgG $(\mathrm{H}+\mathrm{L})$ (red fluorescence) (1:200 in 1\% BSA; Molecular Probes Inc.). Immunofluorescence was visualized using an MRC-1024/2P multiphoton microscope equipped with krypton-argon and Ti-sapphire lasers (Bio-Rad Laboratories, Hercules, California, USA). Photographic images were taken from five random fields.

Rho GTP-binding activity. We measured membraneassociated Rho GTP-binding activity in cultured cardiac myocytes or rat hearts by immunoprecipitating $\left[{ }^{35} \mathrm{~S}\right] \mathrm{GTP}-\gamma \mathrm{S}-\mathrm{labeled}$ Rho proteins using partially purified cardiac myocyte membranes and specific RhoA and Rac1 antibodies (Santa Cruz Biotechnology Inc., Santa Cruz, California, USA) as described (24). Immunoblotting of leftover supernatants indicated that RhoA and Rac1 were completely immunoprecipitated. Nonspecific activity was determined in the presence of excess unlabeled GTP- $\gamma \mathrm{S}(100 \mu \mathrm{M}$; NEN Life Science Products Inc.).

Ferricytochrome c reduction assay. Assay for superoxide anion $\left(0_{2} \cdot{ }^{-}\right)$released into the supernatant was carried out by measuring superoxide dismutase-inhibitable (SOD-inhibitable) reduction of ferricytochrome $c$ as 
described (25). After 24 hours of stimulation in phenol red-free and serum-deficient IT medium, ferricytochrome $c$ was added to the supernatant to a final concentration of $70 \mu \mathrm{M}$ in the presence or absence of SOD $(100 \mathrm{U} / \mathrm{ml})$. Reduction of ferricytochrome $c$ in the supernatant was monitored for 10 minutes at an absorbance of $550 \mathrm{~nm}$ using a spectrophotometer (SPECTRAmax PLUS ${ }^{384}$; Molecular Devices Corp., Sunnyvale, California, USA). Rates of $\mathrm{O}_{2} \cdot{ }^{-}$production were calculated as described (26). The results are expressed as $\mathrm{nmol} / 24 \mathrm{~h} / \mathrm{million}$ cells.

Measurement of $\mathrm{O}_{2}{ }^{-}$production from heart tissues. Intact, nonhomogenized pieces of rat or mouse hearts (100-200 mg) were suspended in a Krebs bicarbonate buffer containing the following reagents, in $\mathrm{mmol} / \mathrm{l}$ : $\mathrm{NaCl}, 118 ; \mathrm{KCl}, 4.7 ; \mathrm{CaCl}_{2}, 1.5 ; \mathrm{MgSO}_{4}, 1.1 ; \mathrm{KH}_{2} \mathrm{PO}_{4}, 1.2$; glucose, 5.6; and $\mathrm{NaHCO}_{3}, 25$. This tissue bath was adjusted to $\mathrm{pH} 7.4$, and gassed with $21 \% \mathrm{O}_{2}$ and $5 \% \mathrm{CO}_{2}$ for 4 hours. The resulting supernatant was used for measuring SOD-inhibitable reduction of ferricytochrome $c$ as described (25). The results were expressed as nmol of $\mathrm{O}_{2}{ }^{-}$produced per gram of cardiac tissue.

Aconitase activity assay. The production of intracellular $\mathrm{O}_{2}{ }^{-}$was determined indirectly by changes in aconitase activity (27). Cultured cells were solubilized in a lysis buffer containing PBS, Triton X-100 (0.2\%), DTPA $(100 \mathrm{mM})$, and citrate $(5 \mathrm{mM})$. Heart tissues were homogenized and resuspended in a buffer containing Tris- $\mathrm{HCl}(50 \mathrm{mM}, \mathrm{pH} 7.6)$, cysteine $(1 \mathrm{mM})$, citrate (1 $\mathrm{mM})$, and $\mathrm{MnCl}_{2}(0.5 \mathrm{mM})$. Cellular extracts $(15 \mu \mathrm{g}$ of protein) were added to a reaction buffer $(0.2 \mathrm{ml})$ containing Tris- $\mathrm{HCl}(50 \mathrm{mM}, \mathrm{pH} 7.4)$, isocitrate $(20 \mathrm{mM})$, and $\mathrm{MnCl}_{2}(0.5 \mathrm{mM})$ at $25^{\circ} \mathrm{C}$, and the formation of cisaconitate from isocitrate was measured spectrophotometrically after 2 minutes, at an absorbance of $240 \mathrm{~nm}$. Aconitase activity was calculated using the extinction coefficient of $3.6 \mathrm{mM}^{-1} \times \mathrm{cm}^{-1}$ and expressed as nmol of cis-aconitate converted $/ \mathrm{min} / \mathrm{mg}$ protein (28).

Animal models of cardiac hypertrophy. We used male Sprague-Dawley rats (8 weeks old, 200-250 g) from Taconic Farms (Germantown, New York, USA). Rats received saline $(25 \mu \mathrm{l} / \mathrm{h})$ or AngII $(200 \mathrm{ng} / \mathrm{kg} / \mathrm{min})$ by osmotic minipumps (ALZET 2002; ALZA Corp., Palo Alto, California, USA), with and without $1.0 \mathrm{ml}$ of preactivated $\operatorname{Sim}(0.2,2$, or $20 \mathrm{mg} / \mathrm{kg} /$ day, subcutaneous) or a corresponding volume of PBS for 14 days. Systolic blood pressure was measured noninvasively using the tail-cuff method (IITC Inc., Woodland Hills, California, USA). Left ventricular (LV) mass, body weight (BW), and serum total cholesterol level were determined for each condition before and after treatment.

For the pressure-overload model, we used male C57BL/ 6 mice (8 weeks old, 20-25 g) from Charles River Laboratories (Wilmington, Massachusetts, USA). Transaortic constriction (TAC) was created using a 7-0 suture tied twice around the aorta and a 27-gauge needle. The needle was then gently retracted, yielding a $60-80 \%$ constriction with an outer aortic diameter of approximately $0.3 \mathrm{~mm}$. Mice received either vehicle or preactivated $\operatorname{Sim}(2 \mathrm{mg} / \mathrm{kg} /$ day, subcutaneous $)$ immediately after surgery. After 4 weeks of TAC, mice were anesthetized with intraperitoneal pentobarbital $(50 \mathrm{mg} / \mathrm{kg})$, and cardiac dimensions and function were analyzed by $15-\mathrm{MHz}$ pulse-wave Doppler echocardiography (SONOS 5500; Hewlett-Packard, Andover, Massachusetts, USA). Systemic arterial blood pressure was measured invasively via the left carotid artery.

\section{Results}

Cell culture. Relatively pure (>95\%) neonatal ventricular myocytes were confirmed by their morphological features using phase-contact microscopy and immunofluorescent staining with a monoclonal anti-cardiac MHC antibody (data not shown). There were no observable adverse effects by AngII, Sim, L-mev, LDL, GGPP, FPP, GGTI-286, FTI-276, C3 TF, PEG-SOD, or PEG-CAT on cellular viability for any treatment conditions.

Statins inbibit AngII-induced cardiac myocyte bypertrophy. Cardiac myocyte hypertrophy is characterized by increased protein content (e.g., $\left[{ }^{3} \mathrm{H}\right]$ leucine uptake) and induction of fetal gene expression (e.g., $A N F$ promoter activity). Treatment with AngII induced neonatal rat cardiac myocyte hypertrophy, as measured by changes in $\left[{ }^{3} \mathrm{H}\right]$ leucine uptake, total protein content, and ANF promoter activity $(50 \% \pm 6 \%, 20 \% \pm 3 \%$, and $180 \pm 20 \%$-fold increases, respectively; $P<0.01$ for all conditions) (Figure 1a). Cotreatment with $\operatorname{Sim}(5 \mu \mathrm{M})$ inhibited the AngII-induced increases in $\left[{ }^{3} \mathrm{H}\right]$ leucine incorporation, total protein content, and ANF promoter activity by $71 \% \pm 4 \%, 85 \% \pm 8 \%$, and $94 \% \pm 5 \%$, respectively. These inhibitory effects of Sim were completely reversed by cotreatment with L-mev or GGPP, but not with FPP or LDL (Figure 1). Treatment with Sim alone, however, did not affect basal $\left[{ }^{3} \mathrm{H}\right]$ leucine incorporation, total protein content, or ANF promoter activity. Similar effects were observed with other statins (e.g., lovastatin, atorvastatin), indicating a class effect of these agents on myocardial HMG CoA reductase.

Using selective inhibitors of geranylgeranylation and farnesylation at their optimal inhibitory concentrations, we found that GGTI-286 $(30 \mu \mathrm{M})$, but not FTI-276 $(20 \mathrm{nM})$, completely blocked AngIIinduced $\left[{ }^{3} \mathrm{H}\right]$ leucine incorporation (Figure $1 \mathrm{~b}$ ). To determine whether inhibition of Rho proteins could be mediating the effects of Sim on cardiac myocyte hypertrophy, Rho proteins were directly inhibited by C3 TF $(50 \mu \mathrm{g} / \mathrm{ml})(29)$. Treatment with C3 TF inhibited the AngII-induced increase in $\left[{ }^{3} \mathrm{H}\right]$ leucine incorporation by $59 \% \pm 5 \%$. In contrast, direct activation of Rho by Escherichia coli cytotoxic necrotizing factor-1 (30) partially reversed the inhibitory effects of Sim (data not shown). Since the Rho protein Rac1 is involved in the generation of reactive oxygen species $(31,32)$ and mediates signaling pathways leading to cardiac hypertrophy (15), we investigated whether superoxide anion $\left(\mathrm{O}_{2} \cdot{ }^{-}\right)$or hydrogen peroxide $\left(\mathrm{H}_{2} \mathrm{O}_{2}\right)$ could be playing a role in AngII-induced cardiac hypertrophy. Cotreatment with cell-permeable 


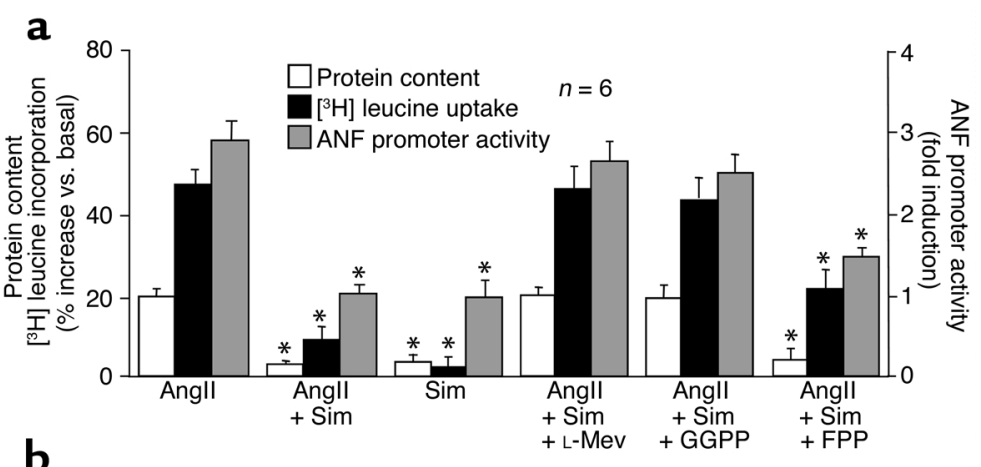

b

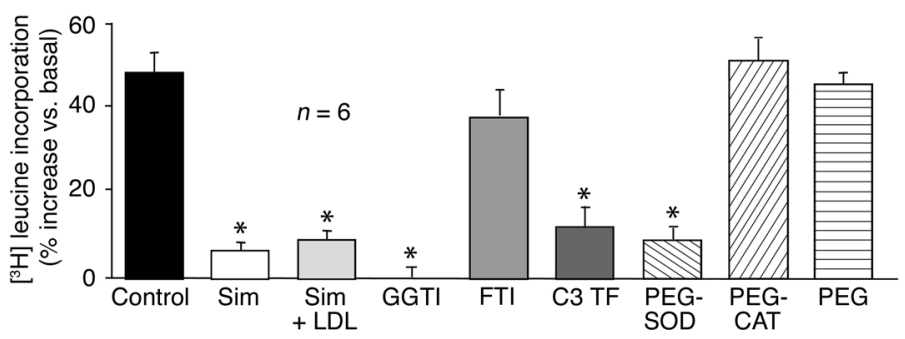

Figure 1

Inhibition of cardiac myocyte hypertrophy by statins. (a) Effects of Angll or Sim (5 $\mu \mathrm{M})$ with and without L-mev, GGPP, or FPP on total protein content, $\left[{ }^{3} \mathrm{H}\right]$ leucine incorporation, and ANF promoter activity. Values are expressed as mean $\pm \mathrm{SEM}$. ${ }^{*} P<0.01$ compared with Angll alone. (b) Effects of $\operatorname{Sim}(5 \mu \mathrm{M})$ with and without LDL, GGTI, FTI, C3 TF, PEG, PEG-SOD, and PEG-CAT on Angll-induced $\left[{ }^{3} \mathrm{H}\right]$ leucine incorporation (Control). Values are expressed as mean \pm SEM. ${ }^{*} P<0.01$ compared with control.

PEG-SOD, but not PEG-CAT or PEG alone, inhibited AngII-induced $\left[{ }^{3} \mathrm{H}\right]$ leucine incorporation (Figure $1 \mathrm{~b})$. These findings suggest that the hypertrophic process is more dependent upon $\mathrm{O}_{2} \cdot{ }^{-}$than on $\mathrm{H}_{2} \mathrm{O}_{2}$.

To determine whether Sim can affect cardiac myocyte cell size and sarcomeric organization, we immunostained cardiac myocytes for two sarcomere-associated proteins, desmin and $\alpha$-actinin. Treatment with AngII induced significant increases in cell size and sarcomere organization (Figure 2a); cotreatment with Sim blocked these AngII-induced cellular changes. The hypertrophic process is also associated with the induction of fetal and structural cardiac genes such as ANF and MLC-2v (4, 33). Indeed, steady-state ANF and MLC- $2 v$ mRNA expression were each increased by about twofold following AngII stimulation $(n=3, P<0.01)$ (Figure 2, $\mathrm{b}$ and c). Cotreatment with Sim inhibited AngII-induced ANF, and to a lesser extent, MLC-2v mRNA expression

\section{Figure 2}

Inhibition of cardiac sarcomere organization, myocyte size, and fetal gene expression by statins. (a) Effects of Angll with and without Sim $(5 \mu \mathrm{M})$ on cardiac myocyte size and sarcomere organization. Double immunofluorescent microscopy was performed using specific antibodies to desmin (upper panel, red color) and $\alpha$-actinin (lower panel, green color). Experiments were performed three times with similar results. Effect of Angll with and without $\operatorname{Sim}(5 \mu \mathrm{M})$ on steady-state (b) ANF and (c) MLC-2v mRNA expression after 24 hours of treatment. Corresponding ethidium bromide-stained $28 \mathrm{~S}$ ribosomal RNA was used to standardize loading. The results shown are representative of three separate experiments. in cardiac myocytes. High basal expression of ANF and MLC- $2 \mathrm{v}$ is attributed to the use of neonatal cardiac myocytes instead of adult cardiac cells. Nevertheless, these findings correlate with the inhibition of cardiac myocyte hypertrophy by Sim.

Inbibition of cardiac bypertrophy by statins is mediated by Rac1 and RhoA. Treatment with AngII increased membrane-associated RhoA and Rac1 GTP-binding activity by $147 \%$ and $87 \%$, respectively $(P<0.05$ for both) (Figure 3a). Cotreatment with Sim completely blocked AngII-induced increases in membrane-associated RhoA and Rac1 GTP-binding activity. These inhibitory effects of Sim were reversed by cotreatment with GGPP, but not FPP, indicating that Sim inhibited the membrane localization and activities of both RhoA and Rac1.

To assess which Rho protein mediates AngII-induced cardiac hypertrophy, we cotransfected rat cardiac myocytes with dominant-negative mutants of Rac1, RhoA, or $\mathrm{Cdc} 42$ and an ANF promoter-luciferase reporter construct. Transfection with dominant-negative Rac1 (N17Rac1), and to a lesser extent, RhoA (N19RhoA), inhibited AngIIinduced $A N F$ promoter activity (Figure 3b). Transfection with $\mathrm{Cdc} 42$ (N17Cdc42) had no inhibitory effect. Cotreatment with Sim further decreased $A N F$ promoter activity in cells transfected with N19RhoA and N17Cdc42, but not N17Rac1. Indeed, N17Rac1 decreased ANF promoter activity to below basal levels $(P<0.05)$. Although cotreatment with GGPP reversed the inhibitory effects of Sim,

a
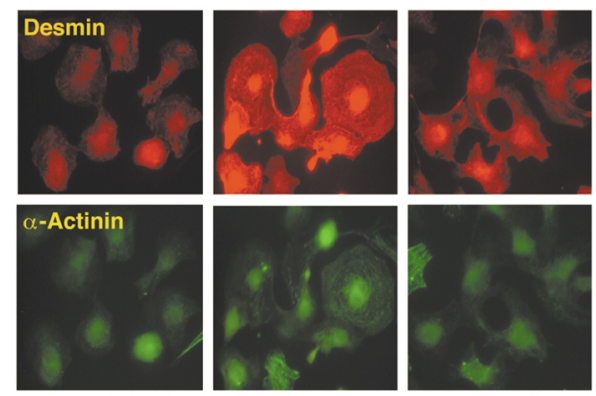

Control

Angll

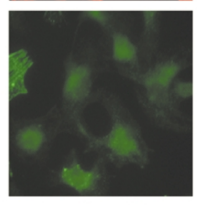

Angll + Sim

b

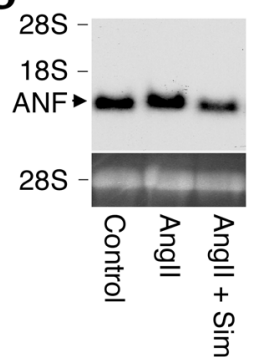

C $28 \mathrm{~S}$ $18 \mathrm{~S}$ MLC-2v $\rightarrow=-$ $28 \mathrm{~S}$





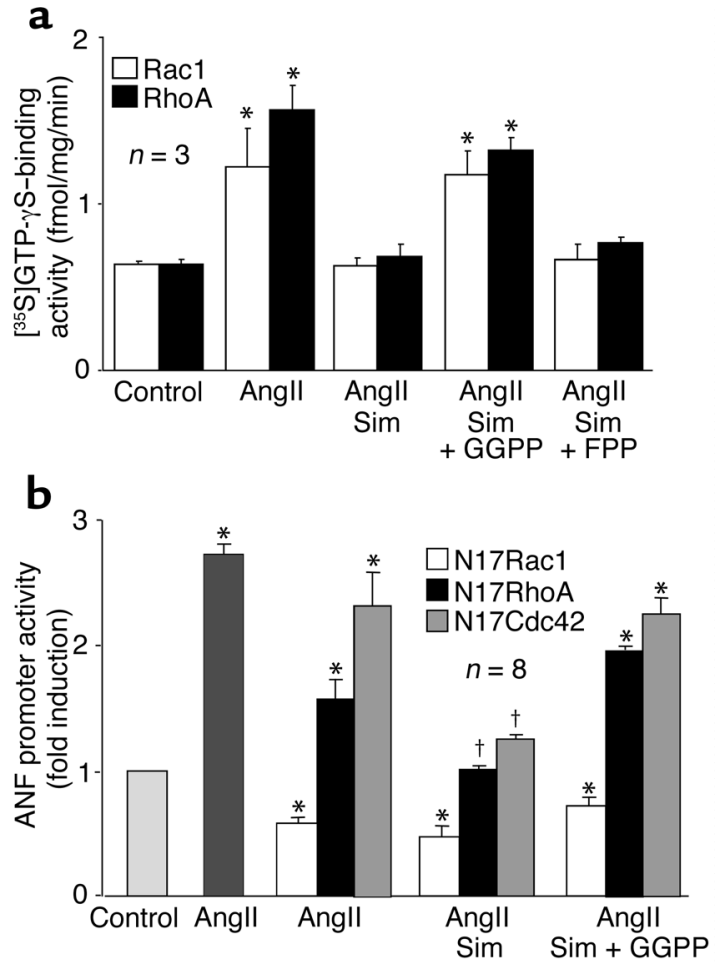

GGPP could not reverse the inhibitory effect of N17Rac1 on ANF promoter activity. These results suggest that Rac1 is the predominant Rho protein that mediates AngII-induced cardiac hypertrophy.

Statins inbibit Rac1-induced $\mathrm{O}_{2}{ }^{-}$production. An important function of Rac in neutrophils (34), fibroblasts (31), and vascular smooth muscle cells (32) is facilitating the assembly of NADPH oxidase, which is a major source of $\mathrm{O}_{2}{ }^{-}$production in these cells. To determine whether the inhibitory effects of Sim on cardiac hypertrophy involve inhibition of $\mathrm{O}_{2} \cdot{ }^{-}$production, we monitored $\mathrm{O}_{2} \cdot{ }^{-}$released into the supernatant by cardiac

\section{Figure 3}

Inhibition of RhoA and Rac1 by statins. (a) Effect of Angll with and without Sim, GGPP, or FPP on membrane-associated Rac1 and RhoA GTP-binding activity in rat cardiac myocytes. Values are expressed as mean \pm SEM. ${ }^{*} P<0.05$ compared with unstimulated cells (Control). (b) Effects of transfection with dominant-negative Rho mutants (N17Rac1, N19RhoA, or N17Cdc42) with and without $\operatorname{Sim}(5 \mu \mathrm{M})$ or GGPP on Angll-induced $A N F$ promoter activity. Values are expressed as mean \pm SEM. ${ }^{*} P<0.01$ compared with transfection with vector alone (Control). $+P<0.05$ compared with Angll and dominant-negative Rho alone. Tx: DN-Rho = N17Rac1, N19RhoA, or N17Cdc42.

myocytes using a ferricytochrome $c$ reduction assay (25). AngII doubled $\mathrm{O}_{2}{ }^{-}$production (Figure 4a). This increase was blocked by cotreatment with $\mathrm{Sim}, \mathrm{C} 3 \mathrm{TF}$, and PEG-SOD (50 U/ml), but not with PEG-CAT (500 $\mathrm{U} / \mathrm{ml}$ ). Treatment with PEG or Sim alone had little or no effect on $\mathrm{O}_{2}{ }^{-}$production. Cotreatment with GGPP completely reversed the inhibitory effect of Sim on $\mathrm{O}_{2} \cdot{ }^{-}$production, but not the effects of $\mathrm{C} 3 \mathrm{TF}$ or PEG-SOD (data not shown).

To confirm that the effects of AngII and $\mathrm{Sim}$ on $\mathrm{O}_{2}{ }^{-}$ production correspond to changes in cellular oxidative stress, we assessed total intracellular oxidation in rat cardiac myocytes by DCF fluorescence. Since $\mathrm{H}_{2} \mathrm{O}_{2}$ is the downstream dismutated product of $\mathrm{O}_{2} \cdot{ }^{-}$, the level of DCF fluorescence, which correlates with increased levels of peroxide-derived reactive intermediates, may also serve as an useful marker for $\mathrm{O}_{2}{ }^{-}$production. Indeed, AngII-induced DCF fluorescence was completely blocked by the addition of PEG-CAT, which converts $\mathrm{H}_{2} \mathrm{O}_{2}$ to $\mathrm{H}_{2} \mathrm{O}$, but not by PEG-SOD, which enhances rather than inhibits the conversion of $\mathrm{O}_{2} \cdot{ }^{-}$to $\mathrm{H}_{2} \mathrm{O}_{2}$ (data not shown). Stimulation with AngII produced a 2.7 -fold increase in intracellular oxidative stress, as measured by DCF fluorescence $(n=3$, $P<0.05$ ) (Figure $4 \mathrm{~b}$ ). This increase was completely

\section{a}

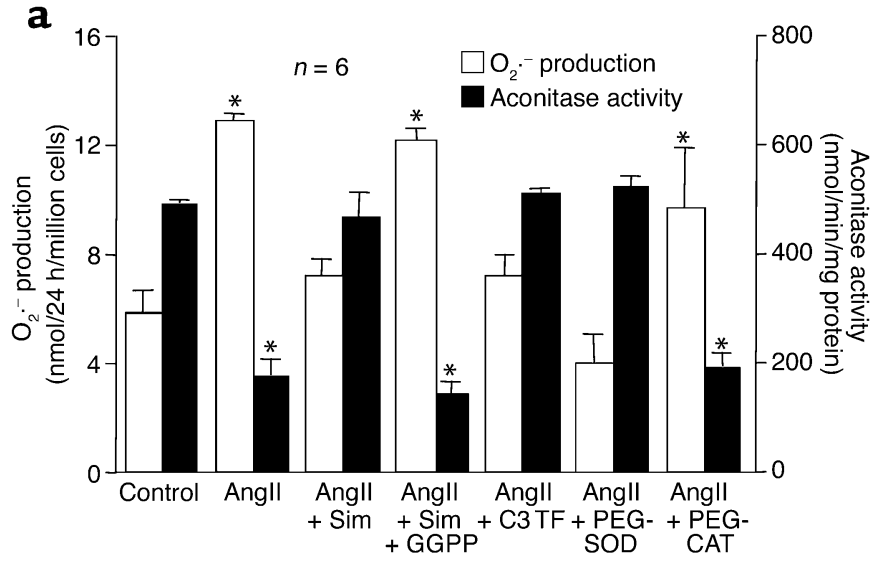

b

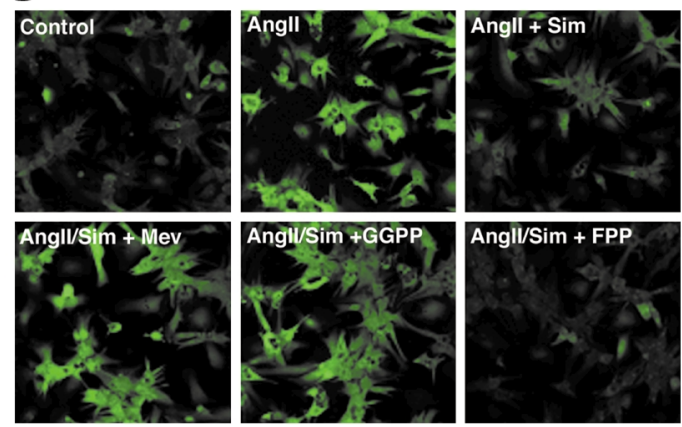

Figure 4

Inhibition of superoxide anion production and intracellular oxidation by statins. (a) Effects of Sim ( $5 \mu \mathrm{M})$, GGPP, C3 TF, PEG-SOD, or PEG-CAT on Angll-induced $\mathrm{O}_{2}{ }^{-}$production in rat cardiac myocytes, as measured by ferricytochrome $c$ reduction and aconitase assays. Values are expressed as mean \pm SEM. ${ }^{*} P<0.01$ compared with unstimulated cells (Control). (b) Effects of Sim $(5 \mu \mathrm{M})$ with and without L-mev, GGPP, or FPP on Angll-induced intracellular oxidation (DCF fluorescence). Results shown were chosen from five random fields and are representative of three separate experiments. 
a

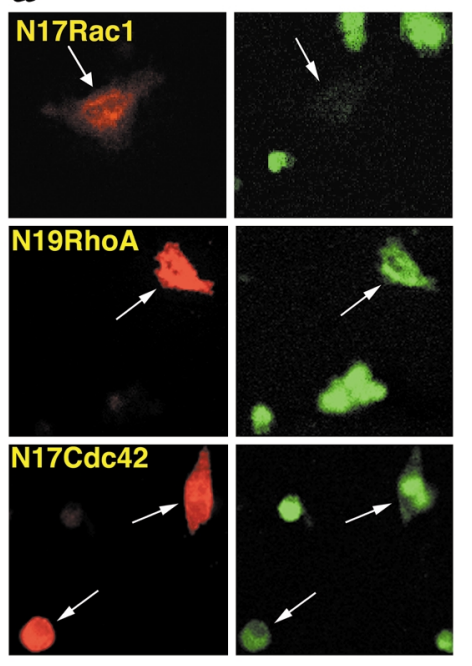

b

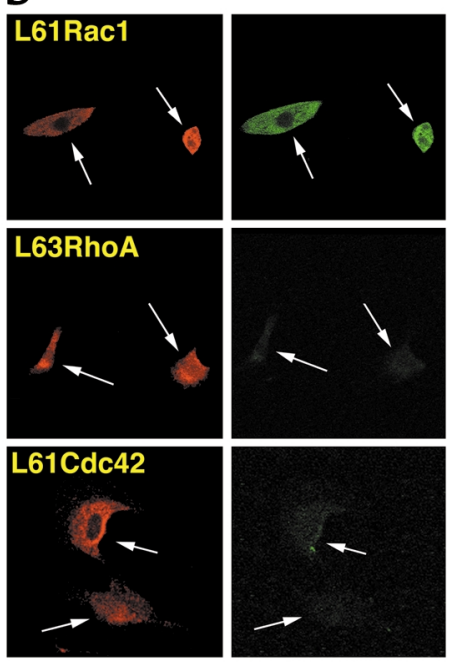

\section{Figure 5}

Inhibition of Rac1-induced intracellular oxidation by statins. Intracellular oxidation in rat cardiac myocytes that were transfected with dominant-negative Rho mutants (N17 or N19) and stimulated with Angll (a), or transfected with constitutively active Rho mutants (L61 or L63) (b). Double fluorescent microscopy was performed to determine the expression of c-myc-tagged Rho mutants (left panel, red color) and corresponding levels of DCF fluorescence (right panel, green color). Experiments were performed three times with similar results. The arrows indicate transfected cardiac myocytes in corresponding panels. blocked by cotreatment with Sim. The inhibitory effect of Sim on intracellular oxidation was reversed by L-mev and GGPP, but not by FPP, suggesting that Rho proteins were mediating the increase in $\mathrm{O}_{2}{ }^{-}{ }^{-}$-derived intracellular oxidation in response to AngII.

Rac1 is required for AngII-induced intracellular oxidation. To determine which Rho proteins mediate the AngIIinduced increase in intracellular oxidation, we transfected rat cardiac myocytes with c-myc-tagged dominant-negative (N17 or N19) or constitutively active (L61 or L63) mutants of Rho, and performed dual fluorescence microscopy for c-myc and intracellular oxidation. Treatment with AngII increased intracellular oxidation, as determined by DCF fluorescence in nontransfected cells or cells transfected with dominantnegative RhoA or Cdc42 (Figure 5a). Transfection with dominant-negative Rac1, however, completely inhibited AngII-induced intracellular oxidation. Conversely, only cells transfected with constitutively active Rac1 showed increased intracellular oxidation; nontransfected cells or cells transfected with constitutively active RhoA or Cdc42 showed no such increase (Figure $5 b$ ). These results indicate that Rac1 mediates AngIIinduced intracellular oxidation, and suggest that Sim inhibits intracellular oxidation by inhibiting Rac1.

Statins inhibit cardiac bypertrophy in vivo. To determine whether our in vitro findings have physiological relevance, we evaluated the effects of statins using two widely used models of cardiac hypertrophy $(35,36)$. AngII infusion caused a substantial increase in systolic blood pressure that was not affected by cotreatment with Sim

\section{Figure 6}

Inhibition of Rac1 and superoxide anion production in rat hearts. Effects of Angll ( $200 \mathrm{ng} / \mathrm{kg} / \mathrm{min}$ ) infusion with and without $\operatorname{Sim}(20$ $\mathrm{mg} / \mathrm{kg} /$ day for 14 days) on (a) membrane-associated Rac1 and RhoA GTP-binding activities and (b) aconitase activity and $\mathrm{O}_{2}{ }^{-}$ released from intact rat hearts. Values are expressed as mean \pm SEM. ${ }^{*} P<0.05$ compared with vehicle-treated animals (Control). ${ }^{\dagger} P<0.05$ compared with animals treated with Angll alone.
(Table 1). Treatment with Sim alone had no effect on systemic blood pressure, BW, or total serum cholesterol levels. AngII infusion (200 ng/kg/day for 14 days) caused a significant increase in LV/BW ratio and LV mass. However, in the short duration of our experimental conditions, AngII did not produce significant deposition of collagen or fibrosis. The increases in LV/BW ratio and LV mass by AngII were inhibited in a concentration-dependent manner by cotreatment with Sim. Similar findings were also observed with atorvastatin, suggesting a class effect on myocardial HMG CoA reductase inhibition.

In hearts from rats treated with AngII infusion, there were increases in membrane-associated RhoA and Rac1 GTP-binding activities, both of which were inhibited by cotreatment with Sim (Figure 6a). Indeed, AngII increased $\mathrm{O}_{2}{ }^{-}$production in intact, nonhomogenized rat heart tissues by $40 \%$ (from $42 \pm 4$ to $59 \pm 5 \mathrm{nmol} / \mathrm{mg}$,

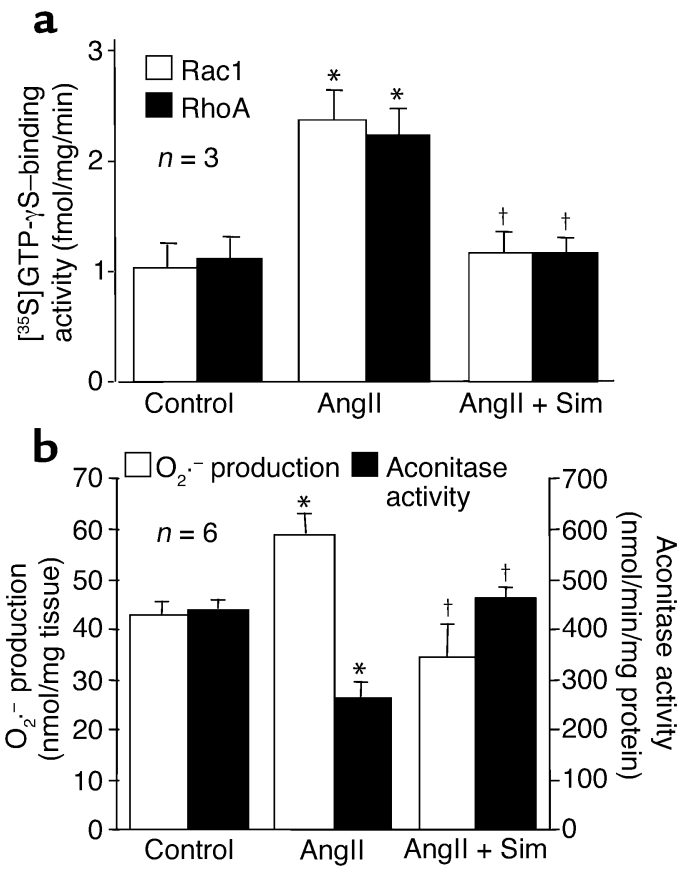


Table 1

Effects of statin on Angll-induced cardiac hypertrophy

\begin{tabular}{|c|c|c|c|c|c|c|}
\hline Parameter & Control & Angll & $\begin{array}{c}\text { Angll + } \\
\operatorname{Sim}(0.2)\end{array}$ & $\begin{array}{l}\text { AnglI + } \\
\text { Sim (2) }\end{array}$ & $\begin{array}{l}\text { AngII + } \\
\text { Sim (20) }\end{array}$ & $\operatorname{Sim}(20)$ \\
\hline Systolic BP $(\mathrm{mmHg})$ & $118 \pm 8$ & $180 \pm 4^{*}$ & $177 \pm 3^{*}$ & $178 \pm 3^{*}$ & $173 \pm 4^{*}$ & $119 \pm 8$ \\
\hline BW (g) & $297 \pm 6$ & $291 \pm 2$ & $289 \pm 6$ & $285 \pm 9$ & $288 \pm 5$ & $287 \pm 7$ \\
\hline LV mass (mg) & $642 \pm 17$ & $826 \pm 23^{*}$ & $781 \pm 16^{*}$ & $707 \pm 26^{* * \dagger}$ & $679 \pm 30^{\dagger}$ & $624 \pm 21^{\dagger}$ \\
\hline $\mathrm{LV} / \mathrm{BW}(\mathrm{mg} / \mathrm{g})$ & $2.16 \pm 0.03$ & $2.84 \pm 0.06^{*}$ & $2.71 \pm 0.07^{*}$ & $2.49 \pm 0.08^{* * \dagger}$ & $2.35 \pm 0.10^{\dagger}$ & $2.17 \pm 0.05^{\dagger}$ \\
\hline $\begin{array}{l}\text { Serum cholesterol } \\
(\mathrm{mg} / \mathrm{dl})\end{array}$ & $125 \pm 9$ & $131 \pm 4$ & $133 \pm 8$ & $129 \pm 5$ & $129 \pm 5$ & $126 \pm 4$ \\
\hline
\end{tabular}

Sim is expressed in $\mathrm{mg} / \mathrm{kg} /$ day. Values are mean $\pm \mathrm{SEM}, n=5-8$ per group. ${ }^{*} P<0.01$ versus control group. ${ }^{*} P<0.05$ versus control group. ${ }^{\dagger} P<0.01$ versus Angll group. BP, blood pressure.

$n=6, P<0.05)$, an effect that was completely blocked by cotreatment with $\operatorname{Sim}(34 \pm 8 \mathrm{nmol} / \mathrm{mg}, n=6, P>0.05$ compared to control) (Figure 6b). These results correlated inversely with a $41 \%$ decrease in aconitase activity in rat heart tissues after AngII infusion (from $440 \pm 27$ to $260 \pm 41 \mathrm{nmol} / \mathrm{min} / \mathrm{mg}, n=6, P<0.005)$, which was completely restored by cotreatment with $\operatorname{Sim}(460 \pm 31$ $\mathrm{nmol} / \mathrm{min} / \mathrm{mg}, n=6, P>0.05$ compared to control). These findings suggest that inhibition of Rho proteins contributed to the statin's inhibitory effect on AngIIinduced $\mathrm{O}_{2}{ }^{-}$production in vivo.

To determine whether the antihypertrophic effect of statins is a central mechanism that could be generalized to other etiologies of cardiac hypertrophy, we investigated the effects of Sim in a pressure-overload model of cardiac hypertrophy. TAC (aortic banding) did not affect systolic blood pressure or BW. However, TAC produced significant increases in the ratio of heart weight (HW) to BW compared with sham-operated mice (Figure 7a). These effects of TAC correlated with a 59\% decrease in aconitase activity in mouse heart tissues (from $490 \pm 22$ to $201 \pm 18 \mathrm{nmol} / \mathrm{min} / \mathrm{mg}, n=3$, $P<0.01)$. Indeed, TAC resulted in anatomical and functional changes that were consistent with cardiac hypertrophy, such as increases in posterior wall thickness and LV end-systolic diameter, and a $25 \%$ decrease in fractional shortening or ejection fraction (Table 2). Cotreatment with Sim attenuated HW/BW ratio by approximately $50 \%$, completely restored aconitase activity in mouse heart tissues, and improved cardiac diastolic and to a lesser extent, systolic function. Furthermore, treatment with Sim significantly decreased cardiac myocyte size, LV wall thickness, and gross cardiac dimensions (Figure 7b).

\section{Figure 7}

Inhibition of pressure overload-induced $\mathrm{O}_{2}{ }^{-}$production and cardiac hypertrophy by statins. (a) Effects of TAC with and without Sim ( $2 \mathrm{mg} / \mathrm{kg} /$ day for 4 weeks) on HW/BW ratio in mice and aconitase activity in mouse hearts. Values are expressed as mean \pm SEM. ${ }^{*} P<0.01$ compared with vehicle-treated sham-operated animals (Sham). ${ }^{\dagger} P<0.05$ compared with TAC treatment without Sim. (b) Cardiac hypertrophy was assessed by hematoxylin and eosin staining of heart tissues (top row; bar $=10 \mu \mathrm{m}$ ) and gross postmortem crosssectional examination of the heart at the level just below the mitral valve (bottom row; bar $=1 \mathrm{~mm}$ ).

\section{Discussion}

Statins prevent the development of cardiac hypertrophy in a cholesterol-independent manner. The mechanism is due in part to the inhibition of isoprenoid synthesis, Rho geranylgeranylation, and Rac1-induced $\mathrm{O}_{2}{ }^{-}$production in cardiac myocytes. Indeed, we found that the nonspecific antioxidant, $\mathrm{N}$-acetylcysteine, was just as effective as statins in inhibiting AngII-induced cardiac hypertrophy (data not shown). However, further studies need to be performed to determine the precise mechanism by which $\mathrm{N}$-acetylcysteine inhibits cardiac hypertrophy, and whether it involves its antioxidant effects. Nevertheless, the ability of statins to decrease $\mathrm{O}_{2}{ }^{-}$production via inhibition of Rac1 suggests that they may have clinical benefits beyond lipid lowering in attenuating the hypertrophic process.

Evidence is emerging that statins and antioxidants could have inhibitory effects on cardiac hypertrophy

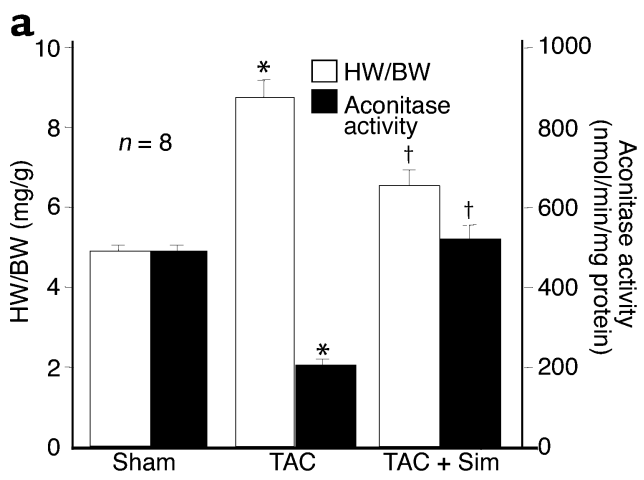

b

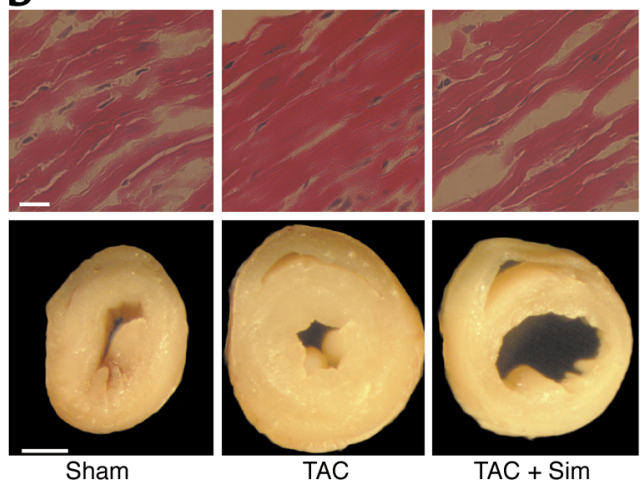


Table 2

Echocardiographic analysis of mice with TAC

\begin{tabular}{lccc}
\hline & Sham & TAC & TAC + Sim \\
Parameter & $117 \pm 2$ & $112 \pm 2$ & $114 \pm 6$ \\
Systolic BP $(\mathrm{mmHg})$ & $0.69 \pm 0.02$ & $1.02 \pm 0.03^{*}$ & $0.84 \pm 0.06^{* * \dagger}$ \\
PWT, diastolic $(\mathrm{mm})$ & $3.27 \pm 0.17$ & $3.44 \pm 0.08$ & $3.27 \pm 0.17$ \\
LVD, diastolic $(\mathrm{mm})$ & $1.95 \pm 0.14$ & $2.38 \pm 0.10^{*}$ & $2.11 \pm 0.13^{\dagger}$ \\
LVD, systolic $(\mathrm{mm})$ & $41 \pm 2$ & $31 \pm 2^{*}$ & $36 \pm 2^{\dagger}$ \\
Fractional shortening $(\%)$ & & &
\end{tabular}

Values are mean $\pm \mathrm{SEM}, n=8$ per group. ${ }^{*} P<0.01$ versus sham group. ${ }^{*} P<0.05$ versus sham group. ${ }^{\dagger} P<0.01$ versus TAC group. PWT, posterior wall thickness; LVD, left ventricular diameter.

(37-39), although the mechanism for these effects remains largely unknown. For example, a recent study showed that statins could inhibit cardiac hypertrophy and fibrosis in a rabbit $\beta$-myosin heavy chain- $\mathrm{Q}^{403}$ mutant model of cardiac hypertrophy without affecting Rac-GTP activity (38). These results suggest that the antihypertrophic effects of statins may not be due to the inhibition of Rho proteins alone, but may also involve the inhibition of other downstream signaling pathways. In contrast to other studies, we did not observe any increase in cardiac fibrosis in our AngII infusion model, probably due to the lower concentration of AngII and the shorter treatment period used in our study. Indeed, cardiac hypertrophy and fibrosis are observed with higher concentrations of AngII (40). Our findings are in agreement with a previous study showing that statins attenuate vascular smooth muscle cell hypertrophy by inhibiting Rac-induced $\mathrm{O}_{2} \cdot{ }^{-}$production (32).

The antihypertrophic effect of statins could also be due, in part, to decreases in AngII type 1 receptor expression (32) or myocardial angiotensin-converting enzyme activity (37). However, the ability of statins to inhibit cardiac myocyte hypertrophy in response to other agonists such as endothelin- 1 and phenylephrine, and to pressure overload, suggests that the predominant mechanism of action includes mechanisms that are distal to the AngII type 1 receptor. Statin therapy also increases vascular nitric oxide production (23), which can potentially decrease blood pressure and attenuate the hypertrophic process. However, there were no significant changes in systemic arterial systolic blood pressure in animals treated with statins in our study. Finally, nitric oxide may also contribute to the direct antihypertrophic effects of statins through favorable effects on cardiac performance and metabolism (41). Further studies with statins in $\mathrm{eNOS}^{-/-}$mice should help clarify these issues.

Statins inhibit the synthesis of important isoprenoid intermediates such as GGPP and FPP, which are important lipid attachments for the posttranslational modification of small GTP-binding proteins such as Rho $(18,19)$. Rho proteins play a critical role in mediating the development of cardiac hypertrophy. For example, RhoA controls the formation of actin stress fiber and focal adhesion complexes through activation of Rho kinases and myosin light chain phosphorylation (42).
Rac1 and Cdc42 regulate actin cytoskeletal processes called lamellipodia (43) and filopodia (44), which may be involved in the morphological changes associated with cardiac hypertrophy. In addition, Rho proteins could also regulate the hypertrophic process by activating downstream signaling molecules such as mitogen-activated protein (MAP) kinases (11, 13). Our finding that overexpression of dominant-negative Rac1 completely inhibited intracellular oxidation and cardiac myocyte hypertrophy, whereas RhoA caused a lesser inhibition and Cdc42 none at all, is in agreement with a previous study showing that Rac1 is required for signal transduction pathways leading to cardiac myocyte hypertrophy (15).

Since the hypertrophic response is a mechanism of physiological adaptation to increases in afterload, it is not entirely clear whether inhibition of the hypertrophic process at the expense of decreasing cardiac performance would actually be beneficial. For example, in experimental models of $\mathrm{LV}$ pressure overload, inhibition of the hypertrophic response leads to the rapid development of dilated cardiomyopathy and heart failure (45). Similar detrimental effects often occur following myocardial infarction, if the hypertrophic process or myocardial remodeling is inhibited (46). However, despite these concerns, our results indicate that statins not only decreased cardiac hypertrophy, but also improved cardiac performance by mostly reducing $L V$ end-diastolic dimensions, even in the presence of persistent elevations in systemic blood pressure and afterload. The precise mechanism(s) underlying these properties of statins is unknown, but it may involve enhancing nitric oxide-mediated improvement in myocardial energy metabolism (41).

In summary, statins are effective agents for preventing the development of cardiac hypertrophy. Of clinical significance is that hypertension, not hypercholesterolemia, is the main risk factor for cardiac hypertrophy (2). These results, therefore, suggest a novel pharmacological approach to treating cardiac hypertrophy in a patient population where statin therapy may not otherwise be indicated. Supporting data from large clinical trials, however, are needed before such recommendations can be made.

\section{Acknowledgments}

We thank Stefan Frantz and Gail K. Adler for technical assistance, and Alan Hall and Wouter Moolenaar for Rho constructs. This work was supported by grants from NIH (HL-52233, HL-62602, and HL-48743, to J.K. Liao), the American Heart Association Bugher Foundation Award (to J.K. Liao), the Banyu-Merck Fellowship in Lipid Metabolism and Atherosclerosis (to M. Takemoto and H. Nakagami), and the Feodor Lynen Fellowship from the Alexander von Humboldt Stiftung (to M. Grimm). 
1. Frohlich, E.D. 1987. Cardiac hypertrophy in hypertension. N. Engl. J. Med. 317:831-833.

2. Vasan, R.S., and Levy, D. 1996. The role of hypertension in the pathogenesis of heart failure. A clinical mechanistic overview. Arch. Intern. Med. 156:1789-1796.

3. Levy, D., Garrison, R.J., Savage, D.D., Kannel, W.B., and Castelli, W.P. 1990 Prognostic implications of echocardiographically determined left ventricular mass in the Framingham Heart Study. N. Engl.J. Med. 322:1561-1566.

4. Chien, K.R., Knowlton, K.U., Zhu, H., and Chien, S. 1991. Regulation of cardiac gene expression during myocardial growth and hypertrophy: molecular studies of an adaptive physiologic response. FASEB J. 5:3037-3046.

5. Shubeita, H.E., Martinson, E.A., Van Bilsen, M., Chien, K.R., and Brown, J.H. 1992. Transcriptional activation of the cardiac myosin light chain 2 and atrial natriuretic factor genes by protein kinase $C$ in neonatal rat ventricular myocytes. Proc. Natl. Acad. Sci. USA. 89:1305-1309.

6. Lembo, G., Hunter, J.J., and Chien, K.R. 1995. Signaling pathways for cardiac growth and hypertrophy. Recent advances and prospects for growth factor therapy. Ann. NY Acad. Sci. 752:115-127.

7. Dorn, G.W., and Brown, J.H. 1999. Gq signaling in cardiac adaptation and maladaptation. Trends Cardiovasc. Med. 9:26-34.

8. Mende, U., et al. 1998. Transient cardiac expression of constitutively active Galphaq leads to hypertrophy and dilated cardiomyopathy by calcineurin-dependent and independent pathways. Proc. Natl. Acad. Sci. USA. 95:13893-13898

9. Thorburn, A., et al. 1993. HRas-dependent pathways can activate morphological and genetic markers of cardiac muscle cell hypertrophy. J. Biol. Chem. 268:2244-2249.

10. Aoki, H., Izumo, S., and Sadoshima, J. 1998. Angiotensin II activates RhoA in cardiac myocytes: a critical role of RhoA in angiotensin IIinduced premyofibril formation. Circ. Res. 82:666-676.

11. Sah, V.P., Hoshijima, M., Chien, K.R., and Brown, J.H. 1996. Rho is required for Galphaq and alpha1-adrenergic receptor signaling in cardiomyocytes. Dissociation of Ras and Rho pathways. J. Biol. Chem. 271:31185-31190.

12. Van Aelst, L., and D’Souza-Schorey, C. 1997. Rho GTPases and signaling networks. Genes Dev. 11:2295-2322.

13. Hoshijima, M., Sah, V.P., Wang, Y., Chien, K.R., and Brown, J.H. 1998. The low molecular weight GTPase Rho regulates myofibril formation and organization in neonatal rat ventricular myocytes. Involvement of Rho kinase. J. Biol. Chem. 273:7725-7730.

14. Sah, V.P., et al. 1999. Cardiac-specific overexpression of RhoA results in sinus and atrioventricular nodal dysfunction and contractile failure. $J$ Clin. Invest. 103:1627-1634

15. Pracyk, J.B., et al. 1998. A requirement for the rac1 GTPase in the signal transduction pathway leading to cardiac myocyte hypertrophy. J. Clin. Invest. 102:929-937.

16. 1994. Randomised trial of cholesterol lowering in 4444 patients with coronary heart disease: the Scandinavian Simvastatin Survival Study (4S). Lancet. 344:1383-1389.

17. Shepherd, J., et al. 1995. Prevention of coronary heart disease with pravastatin in men with hypercholesterolemia. West of Scotland Coronary Prevention Study Group. N. Engl. J. Med. 333:1301-1307.

18. Goldstein, J.L., and Brown, M.S. 1990. Regulation of the mevalonate pathway. Nature. 343:425-430.

19. Casey, P.J. 1995. Protein lipidation in cell signaling. Science. 268:221-225.

20. Springhorn, J.P., and Claycomb, W.C. 1989. Preproenkephalin mRNA expression in developing rat heart and in cultured ventricular cardiac muscle cells. Biochem. J. 258:73-78.

21. Libby, P. 1984. Long-term culture of contractile mammalian heart cells in a defined serum-free medium that limits non-muscle cell proliferation. J. Mol. Cell. Cardiol. 16:803-811.

22. Calderone, A., Thaik, C.M., Takahashi, N., Chang, D.L.F., and Colucci, W.S. 1998. Nitric oxide, atrial natriuretic peptide, and cyclic GMP inhibit the growth-promoting effects of norepinephrine in cardiac myocytes and fibroblasts. J. Clin. Invest. 101:812-818.

23. Laufs, U., La Fata, V., Plutzky, J., and Liao, J.K. 1998. Upregulation of endothelial nitric oxide synthase by HMG CoA reductase inhibitors. Circulation. 97:1129-1135
24. Laufs, U., and Liao, J.K. 1998. Post-transcriptional regulation of endothelial nitric oxide synthase mRNA stability by Rho GTPase. J. Biol. Chem 273:24266-24271.

25. Matsubara, T., and Ziff, M. 1986. Increased superoxide anion release from human endothelial cells in response to cytokines. J. Immunol. 137:3295-3298.

26. Ohara, Y., Peterson, T.E., and Harrison, D.G. 1993. Hypercholesterolemia increases endothelial superoxide anion production. J. Clin. Invest. 91:2546-2551.

27. Gardner, P.R., and Fridovich, I. 1992. Inactivation-reactivation of aconitase in Escherichia coli. A sensitive measure of superoxide radical. J. Biol. Chem. 267:8757-8763.

28. Kennedy, M.C., Emptage, M.H., Dreyer, J.L., and Beinert, H. 1983. The role of iron in the activation-inactivation of aconitase. J. Biol. Chem. 258:11098-11105.

29. Fritz, G., and Aktories, K. 1994. ADP-ribosylation of Rho proteins by Clostridium botulinum exoenzyme C 3 is influenced by phosphorylation of Rho-associated factors. Biochem. J. 300:133-139.

30. Aktories, K. 1997. Bacterial toxins that target Rho proteins. J. Clin. Invest. 99:827-829.

31. Sundaresan, M., et al. 1996. Regulation of reactive-oxygen-species generation in fibroblasts by Rac1. Biochem. J. 318:379-382.

32. Wassmann, S., et al. 2001. Inhibition of geranylgeranylation reduces angiotensin II-mediated free radical production in vascular smooth muscle cells: involvement of angiotensin AT1 receptor expression and Rac1 GTPase. Mol. Pharmacol. 59:646-654.

33. Knowlton, K.U., et al. 1991. Co-regulation of the atrial natriuretic factor and cardiac myosin light chain-2 genes during alpha-adrenergic stimulation of neonatal rat ventricular cells. Identification of cis sequences within an embryonic and a constitutive contractile protein gene which mediate inducible expression. J. Biol. Chem. 266:7759-7768.

34. Knaus, U.G., Heyworth, P.G., Evans, T., Curnutte, J.T., and Bokoch, G.M. 1991. Regulation of phagocyte oxygen radical production by the GTPbinding protein Rac 2. Science. 254:1512-1515.

35. Harada, K., et al. 1998. Pressure overload induces cardiac hypertrophy in angiotensin II type $1 \mathrm{~A}$ receptor knockout mice. Circulation. 97:1952-1959.

36. Kim, S., et al. 1995. Angiotensin II induces cardiac phenotypic modulation and remodeling in vivo in rats. Hypertension. 25:1252-1259.

37. Luo, J.D., Zhang, W.W., Zhang, G.P., Guan, J.X., and Chen, X. 1999. Simvastatin inhibits cardiac hypertrophy and angiotensin-converting enzyme activity in rats with aortic stenosis. Clin. Exp. Pharmacol. Physiol. 26:903-908.

38. Patel, R., et al. 2001. Simvastatin induces regression of cardiac hypertrophy and fibrosis and improves cardiac function in a transgenic rabbit model of human hypertrophic cardiomyopathy. Circulation. 104:r27-r34.

39. Nakamura, K., et al. 1998. Inhibitory effects of antioxidants on neonatal rat cardiac myocyte hypertrophy induced by tumor necrosis factoralpha and angiotensin II. Circulation. 98:794-799.

40. Ramires, F.J., Sun, Y., and Weber, K.T. 1998. Myocardial fibrosis associated with aldosterone or angiotensin II administration: attenuation by calcium channel blockade. J. Mol. Cell. Cardiol. 30:475-483.

41. Recchia, F.A., et al. 1999. Nitric oxide controls cardiac substrate utilization in the conscious dog. Cardiovasc. Res. 44:325-332.

42. Kimura, K., et al. 1996. Regulation of myosin phosphatase by Rho and Rho-associated kinase (Rho-kinase). Science. 273:245-248.

43. Ridley, A.J., Paterson, H.F., Johnston, C.L., Diekmann, D., and Hall, A. 1992. The small GTP-binding protein rac regulates growth factorinduced membrane ruffling. Cell. 70:401-410.

44. Nobes, C.D., and Hall, A. 1995. Rho, rac, and cdc42 GTPases regulate the assembly of multimolecular focal complexes associated with actin stress fibers, lamellipodia, and filopodia. Cell. 81:53-62.

45. Rogers, J.H., et al. 1999. RGS4 causes increased mortality and reduced cardiac hypertrophy in response to pressure overload. J. Clin. Invest. 104:567-576

46. Sutton, M.G., and Sharpe, N. 2000. Left ventricular remodeling after myocardial infarction: pathophysiology and therapy. Circulation. 101:2981-2988 